\title{
THE SUMMER MEETING IN MADISON
}

The seventy-third summer meeting of the American Mathematical Society was held in Madison, Wisconsin, from Tuesday through Friday, August 27-30, 1968. All sessions were held in lecture rooms and classrooms of the University of Wisconsin. There were 2,029 persons in attendance, including 1,036 members of the Society. This meeting of the Society was held in conjunction with meetings of the Mathematical Association of America, the Society for Industrial and Applied Mathematics, the Institute for Mathematical Statistics, and $\mathrm{Pi} \mathrm{Mu}$ Epsilon.

There were two sets of Colloquium Lectures each consisting of four lectures. Professor Donald C. Spencer of Stanford University spoke on the subject "Overdetermined systems of partial differential equations." Professor Spencer was introduced at his four lectures by Professor Charles B. Morrey, Jr., Professor Victor W. Guillemin, and Professor Kennan T. Smith (twice). The other Colloquium Lecturer was Professor John W. Milnor of Princeton University, the University of California, Los Angeles, and the Massachusetts Institute of Technology. His topic was "Uses of the fundamental group." The presiding officers at Professor Milnor's four lectures were Professor Charles B. Morrey, Jr., Professor Samuel Eilenberg, Professor Deane Montgomery, and Professor Norman E. Steenrod.

By invitation of the Committee to Select Hour Speakers for Annual and Summer Meetings, there were three invited addresses. Professor Wu-Chung Hsiang of Yale University, who was introduced by Professor Franklin P. Peterson, spoke on "Nonsimply connected differential topology." Professor Phillip A. Griffith of the University of Houston and Princeton University addressed the society on the topic "Some transcendental problems in algebraic geometry." $\mathrm{He}$ was introduced by Professor Donald C. Spencer. The third speaker was Professor Victor W. Guillemin of the Massachusetts Institute of Technology. He was also introduced by Professor Donald C. Spencer and spoke on "Recent developments in the theory of pseudogroups."

There were thirty-two sessions for the presentation of contributed papers. The following members of the Society presided: Richard A. Askey, Bruce C. Berndt, G. Robert Blakely, C. Edmund Burgess, Kuo-Tsai Chen, Y. W. Chen, Harold G. Diamond, Matthew P. Gaffney, Andrew M. Gleason, Emil Grosswald, Paul R. Halmos, Simon Hellerstein, Frederick Hoffman, Meyer Jerison, Nicholas D. Kazarinoff, John D. Kiltinen, Ralph M. Krause, Lawrence S. Levy, Leon R. McCulloh, Donald L. McQuillan, Joseph B. Muskat, How- 
ard A. Osborn, Walter V. Philipp, G. Baley Price, Lee A. Rubel, Mary Ellen Rudin, Allen L. Shields, Michael Voichick, Wolfgang R. Wasow, Paul M. Weichsel, Chung-Tao Yang, Joseph A. Zilber.

The Council met on Tuesday, August 27 and at an adjourned meeting on the next day. The Secretary announced that the following 341 persons had been elected to ordinary membership:

Aksen, Howard S., Blaisdell Publishing Company, Waltham, Massachusetts;

Aldridge, Anne G., University of California, Santa Barbara;

Alquiza, Marcelo, Mindanao State University, Marawi City, Philippines;

Amsbury, Wayne P., Union Carbide Corporation, Oak Ridge, Tennessee;

Anderson, Donald G. M., Harvard University;

Andesman, Peter, 4 Split Tree Road, Scarsdale, New York;

Aramburu, Juan C., University of Alabama-Huntsville;

Babakhanian, Ararat, University of Illinois;

Bacon, Donald A., International Business Machines Corporation, Cambridge, Massachusetts;

Bacon, Donald C. Jr., National Aeronautical and Space Administration, Edwards Air Force Base, California;

Bajaj, Prem N., Case Western Reserve University;

Balas, Egon, Carnegie-Mellon University;

Barbee, W. Louise, Eastern Michigan University;

Barnes, Bernis A., Jackson State College;

Bartel, Gladwin E., Whitworth College;

Barton, James D., University of Tennessee;

Beal, Roy R., International Business Machines Corporation, Cleveland, Ohio;

Beavan, Robert B., US Navy, San Francisco, California;

Benedict, Brother J., De La Salle College, Manila, Philippines;

Berkman, Robert A., University of Illinois, Urbana;

Berney, Ernest S., Arizona State University;

Berresford, Geoffrey C., New York University-Courant;

Birmingham, Robert L., Indiana University;

Blank, Samuel J., Northeastern University;

Booth, George W., Brooklyn College (CUNY);

Borchers, Hans-Jurgen, University of Gottingen, Gottingen, Germany;

Borchers, Raymond V., National Aeronautical and Space Administration, Greenbelt, Maryland;

Bradley, Joe Carroll, Westinghouse, Baltimore, Maryland;

Brady, Stephen W., Wichita State University;

Briggs, Barbara H., University of Houston;

Brooks, Thomas H., Mitre Corporation, Bedford, Massachusetts;

Brown, John K., Ohio State University;

Browning, Ronald Lee, New York State University, College at Geneseo;

Bunge, Marta C., McGill University;

Cardone, Joseph M., Alabama College;

Carreau, Francois, University of Montreal;

Casciotti, Leonard A., PMC Colleges;

Caviness, B. F., Duke University;

Celebiler, Mehmeti, University of Pennsylvania;

Chaffman, Louis F., Alextronics Corporation, Baltimore, Maryland; 
Chalmers, Bruce L., University of California, Riverside;

Champagne, Carol Z., Factory Mutual Engineering Association, Norwood, Mas. sachusetts;

Chase, Warren E., Worcester Polytechnic Institute;

Chhon, Has, Minister of the Interior, Phnom Penh, Cambodia;

Chidester, Connie G., Upjohn Company, Kalamazoo, Michigan;

Chu, Chinku, Choong Nam University, Tae Jon, Korea;

Citron, Richard I., Illinois Institute of Technology;

Cohen, Rina E., Waterloo University;

Cole, Henry Samuel, West Coast University;

Connors, Ronald B., Roosevelt Hospital, New York, New York;

Cook, Charles K., Tri-State College;

Cook, David E., University of Mississippi;

Crowell, Joseph S., Union Carbide Corporation, Oak Ridge, Tennessee;

Davies, Margaret Hilary, University of Calgary;

Davis, Harry F., University of Waterloo;

Davis, Henry W., University of New Mexico;

Debney, George C. Jr., TRW Systems, Houston, Texas;

Demeyer, Frank R., Purdue University;

Deruaz, Marcel A. F., University of Ottawa;

DeStefano, Frank, Hewlett High School, Hewlett, New York;

Detry, Ronald J., Illinois Institute of Technology;

Dewart, Alan C., California State, Division of Highways, San Francisco, California;

Dey, Manoj K., Netarhat Public School, Bihar, India;

Doede, Robert W., University of Pennsylvania;

Dowling, John I., St. Dungstan's University;

Dudley, Underwood, DePauw University;

Duggan, Edward Ignatius (Bro.), Iona College;

Dunkel, Gregory M., National Biomedical Research Foundation, Silver Spring, Maryland;

Dupont, Judy S., 2348 Sheridan, Houston, Texas;

Epasinghe, Piyadasa W., Vidyodaya University of Ceylon, Nugegoda, Ceylon;

Epstein, David I., Northeastern University;

Erle, Dieter H. (Dr.), University of Bonn, Bonn, Germany;

Espelie, Mary S., University of Maryland;

Estes, Dennis R., California Institute of Technology;

Eum, Sang-Seup, Sung Kyun Kwan University, Seoul, Korea;

Fenyo, Istvan, Math. Inst. der Universitat, Rostock, East Germany;

Fleckner, Oscar L. Jr., Ohio State University;

Fleming, Walter, Hamline University;

Freiman, Richard, University of Maryland, Baltimore, Maryland;

Friesen, Donald Kent, University of Illinois, Urbana;

Fuller, Richard V., University of North Carolina at Charlotte;

Garcia, Victor M., University of Puerto Rico, Rio Piedras;

Gavala, George W., 560 N. 4th Avenue, Sharon, Pennsylvania;

Girard, Dennis M., Ohio State University;

Gittleman, Arthur P., California State College at Long Beach;

Gold, Ira, Elder House, 175 W. Knox, Galesburg, Illinois;

Goldstein, Charles I., Brookhaven National Laboratories, Upton, New York;

Goldstein, Herbert, Columbia University; 
Good, James C., University of California, Davis;

Grafton, Robert B., University of Missouri;

Green, John W., University of Oklahoma;

Green, Steven John, Philco-Ford, Houston, Texas;

Greenwald, Ruth N., Fundamental Methods Associates, New York, New York;

Gunatilake, Patikirige D., University of Colombo, Colombo, Ceylon;

Hack, Melvin N., Argonne National Laboratory, Argonne, Illinois;

Hall, Japheth Jr., Stillman College;

Hardy, John W. Jr., University of Texas;

Harris, John Hollister, Owen College;

Harrow, Martin, Sir George Williams University;

Harty, Sister M. Raymond C., Edgewood College;

Haynes, Michael J., Alma College;

Hausen, Jutta, New Mexico State University;

Hebert, D. J. Jr., University of Texas;

Heble, Madhav P., University of Toronto;

Heinold, Robert D., Raytheon Company, Bedford, Massachusetts;

Henderleiter, William M., University of Wisconsin-Sheboygan;

Henschen, Lawrence Joseph, University of Illinois, Urbana;

Herbold, Robert J., Procter and Gamble Company, Cincinnati, Ohio;

Hightower, James K., California State Polytechnic College;

Hjalmarson, Gordon R., Houghton Mifflin Company, Boston, Massachusetts;

Hodges, Wilfrid A., University of California, Los Angeles;

Hofmann, Charles E. III, LaSalle College;

Hong, Sung Sa, Sogang College, Seoul, Korea;

Houde, Richard A., Assumption College;

Houston, Johnny L., Stillman College;

Howes, Norman R., Texas Instruments, Richardson, Texas;

Hsieh, Hsiao-Chung, University of California, Irvine;

Hubert, John J., University of Alberta;

Hurt, Norman E., New York University, Courant Institute of Mathematical Sciences;

Hurt, James J., University of Iowa;

Hussain, Ansar, British American Oil Company of Canada, Toronto, Ontario, Canada;

Jackins, Timothy H., Stanford University;

Jacobson, Richard A., Houghton College;

Jamet, Pierre, Commissioner of Energy Atomic, Villeneuve St., George, France;

Jenkyns, Thomas A., University of Waterloo;

Jensen, Carol N., Susquehanna University;

Jensen, Lawrence P., Illinois Institute of Technology;

Jerri, Abdul Jabbar, Clarkson College of Technology;

Johnson, Dommie C., McDonnell-Douglas Aircraft Corporation, Huntington Beach, California;

Johnson, Lee W., Virginia Polytechnic Institute;

Johnson, Vivian M., 227 W. Norrie Street, Ironwood, Michigan;

Jones, Ralph, University of Wisconsin, Madison;

Jones, Susan H., University of California, Davis;

Jordan, F. James., University of North Carolina;

Justice, James H., University of Maryland, College of Arts and Sciences;

Justice, Jay Vernon, University of Texas; 
Kahane, Charles S., University of Minnesota;

Kammler, Daniel A., Southern Illinois University;

Katz, Nicholas M., Princeton University;

Kerlin, John E. Jr., University of California, Irvine;

Kilgore, Robert B., University of Pittsburgh;

Kim, Joong Ho, University of South Carolina;

Kim, Woo Jong, Carnegie-Mellon University;

Kleitman, Daniel J., Massachusetts Institute of Technology;

Klimko, Lawrence A., Ohio State University;

Knutson, Gerhard Walter, Michigan State University;

Koh, Eusebio L., University of South Carolina;

Korsan, Robert James, Westinghouse, Churchill Boro, Pennsylvania;

Lough, Paul F. (Lt.), US Air Force, Scott Air Force Base, Illinois;

Kramer, Earl S., University of Michigan;

Krogh, Fred T., TRW System, Hermosa Beach, California;

Kunofsky, Judith, University of California, Los Angeles;

Kuntz, Richard A., University of Maryland;

Landweber, Lawrence H., University of Wisconsin, Madison;

Latil, Bertrand, Western Michigan University;

Lau, Kar-Wang, University of South Carolina;

Laudenbach, Francois, CNRS, Center de Mathematiques de l'Ecole Polytechnique, Paris, France;

Lazar, Aldo J., University of Washington;

Lazar, David I., Temple University;

Lea, James D., Esso Production Research Company, Houston, Texas;

Lee, Eugene T. Y., University of California, La Jolla;

Lee, Jhong S., George Washington University;

Lee, Joseph Han-Tack, Sogang Jesuit College, Seoul, Korea;

Leroux, Pierre, University of Montreal;

Levko, John J., Lehigh University;

Liffring, Robert F., United States Army, Ft. McClellan, Alabama;

Lipow, Peter R., University of Wisconsin;

Liu, Jong-Ding, Academia Sinica, Nankang (Taiwan), China;

Lorentz, Philip J., Friends Seminary, New York, New York;

Lurye, Jerome R., TRG Incorporated, Melville, New York;

Lutz, James Alan, University of Maryland;

Manes, Ernest G., Harvey Mudd College;

Manke, Arno E., Missouri Valley College;

Marlin, Joe A., North Carolina State University at Raleigh;

Marsten, Roy E., McDonnell-Douglas Aircraft Company, Santa Monica, California;

Martin, Benjamin J., Purdue University;

Massagli, Robert A., Central Washington State College;

Mathews, John H., Michigan State University;

Mather, Michael R., Rice University;

Matkowsky, Bernard J., Rensselaer Polytechnic Institute;

Maulucci, Ruth Anne, University of Massachusetts;

Mayer, Meinhard E., University of California, Irvine;

McAlpin, John H., University of Colorado;

McCabe, Bernard J., Bellcomm Incorporated, Washington, D. C.;

McLaughlin, Eileen, State University of New York, College at Potsdam; 
Meek, James L., University of Arkansas;

Merryman, Emory H., University of Georgia;

Meskin, Stephen A., City University of New York;

Meslans, Denis L., St. Louis University;

Miller, John G., University of California, Los Angeles;

Minassian, Donald P., Butler University;

Misner, John, University of Southern California;

Montgomery, Richard G., Clark University;

Morales, Manuel J., Instituto Pedagogico Experimental, Barquisimeto, Venezuela;

Morey, Philip S. Jr., University of Omaha;

Moro, Antonio, University of Florence, Florence, Italy;

Mudholkar, Govind S., University of Rochester;

Mullin, Ronald C., University of Waterloo;

Murray, Ruth M., College of Du Page;

Myhill, John, State University of New York at Buffalo;

Myung, Hyo Chul, Michigan State University;

Napoli, Thomas J., DeVry Technical Institute;

Newton, Theodore D., University of Guelph;

Nigam, Lakshmi N., Indian Institute of Technology, Bombay, India;

Oh, Hi-Jun, Choong Nam University, Tae Jon, Korea;

Oliver, Carl E., University of Alabama;

Osborne, Dick, San Diego State College;

Oum, Jang I., Pusan National University, Pusan, Korea;

Padmanabhan, Appaswamy R., Indiana University;

Pao, Juliet Zue (Mrs.), University of Alabama in Huntsville;

Papadakis, John S., Brooklyn Polytechnic Institute;

Paradis, Andre, College of Maisonneuve;

Parham, Arthur R. Jr., Aurora College;

Park, Kyung C., Seoul National University, Seoul, Korea;

Pasiencier, Samuel, New York University-Courant;

Payne, Thomas H., University of California, Riverside;

Peiffer, Barry L., Susquehanna University;

Penaranda, Pedro S., Lane College;

Perrink Francois M., 1 Place A Jutard, Lyon, France;

Pertica, Horacio N., IBM, World Trade, White Plains, New York;

Pfeiffer, Isaac R., McGill University;

Pflugtelder, Hala O., Temple University;

Phillips, J. Richard, University of Illinois;

Pineau, Gerald W., St. Dungstan's University;

Porter, John R., University of Pittsburgh;

Portinari, Joao Candido, Pontificia University Catolica, Rio de Janeiro, Brazil;

Powers, Charles B., Del Mar College;

Powers, Michael J., Indiana State University;

Rabina, Manuel J., Alvernia College;

Rabinowitz, Paul H., Stanford University;

Ramey, Charles E., HRB-Singer Incorporated, Ft. Walton Beach, Florida;

Reynolds, Gary Hill, University of California, Berkeley;

Richardson, Daniel J., Wisconsin State University, Oshkosh;

Richardson, Robert L., Appalachian State University;

Richardson, William H., Wichita State University; 
Riley, Charles A., R. D. No. 1, Keene, New Hampshire;

Rim, Jeong D., Yonsei University, Seoul, Korea;

Rivera-Muniz, Pedro Ivan, Grand Valley State College;

Robinson, Joanna Ryten (Mrs.), Bishop College;

Rochon, Lloyd G., Chabot College;

Rodriguez, Dennis M., University of California, Riverside;

Roeling, Lloyd G., Rice University;

Rogers, Thomas De F. Jr., University of Alberta;

Rose, G., Oregon State University;

Rosenblum, Lawrence J., Ohio State University;

Rosenfeld, Moshe, University of Washington;

Rosenthal, Paul L., Stanford University;

Rudolph, Luther D., Syracuse University;

Rueve, Charles R., St. Joseph's College;

Ruoff, Dieter, University of Manitoba;

Rutledge, William A., Old Dominion College;

Sadr, Mohammad T., North Dakota State University;

Samaranayake, V. K., University of Colombo, Colombo, Ceylon;

Sanders, Timothy D., Occidental College;

Sardinas, August A., Villanova University;

Saywer, Jane O., Virginia Polytechnic Institute;

Schmidt, Roland, University of Frankfurt, Frankfurt, Germany:

Schoenwaelder, Ulrich F., University of Frankfurt, Frankfurt, Germany;

Schwartz, Lawrence E., University of Rochester;

Selzer, Adolph, United States Navy, New London, Connecticut;

Sen, Dipak K., University of Toronto;

Shachtman, Richard H., University of Maryland;

Shapiro, Louis W., Howard University;

Shen, Chung Yi, Simon Fraser University;

Shepard, C. D., University of Michigan;

Shilkret, Niel, Polytechnic Institute of Brooklyn;

Shivakumar, Pappur N., Karnatak University, Karnatak, India;

Shklov, Nathan, University of Windsor;

Shoemaker, Edward L., 108 Hedy Lane, Davis, California;

Shumway, David G., Case Western Reserve University;

Shumway, Richard J., University of Minnesota;

Siddalingaiah, Honnappa, Siddaganga Institute of Technology, Tumkur, India;

Sidman, Robert D., University of Connecticut;

Silverman, Roy S., University of British Columbia;

Simmons, Harold F., California State Polytechnic College;

Smith, Kempton J. C., University of North Carolina, Chapel Hill;

Smith, Roberta R., Lockheed Palo Alto Research Laboratory, Palo Alto, California;

Somerville, Paul N., Florida Institute of Technology;

Sparkes, Harry P. Jr., 557 Chapala Drive, Pacific Palisades, California;

Spence, Lawrence E., Michigan State University;

Spezeski, William J., State College at Worcester;

Stafford, Raymond A., University of Tennessee;

Stenger, Frank, University of Michigan;

Stephens, Larry M., DeVry Technical Institute;

Stern, Frederick, Stevens Institute of Technology; 
Stern, James Leroy, University of Idaho;

Stevens, Charles M., 2218 S. Wallace Avenue, Chicago, Illinois;

Stouffer, Robert Thomas, University of New Mexico;

Stratopoulos, George M., Weber State College;

Suen, Robert, St. Dunstan's University;

Suhadolnik, Michael F., Frostburg State College;

Sward, Marcia P., 2825 Greenvale Street, Chevy Chase, Maryland;

Sweet, Roland A., Cornell University;

Swerdlove, Linda S., LeMoyne College;

Szego, Peter A., Ampex, Redwood City, California;

Tabibzadeh, Fariborz, Burroughs Corporation, Chicago, Illinois;

Tahara, Clyde T., Barber-Scotia College;

Taucci, E. Barbara, 454 Pursell Street, Phillipsburg, New Jersey;

Tench, Alan Howard, University of Colorado;

Terrell, Thomas R., Ohio State University;

Terrier, Jean Marc, University of Montreal;

Tolentino, Aristides J., Notre Dame Colleges, Cotabato City, Philippines;

Tomiyama, Jun, Yamagata University, Yamagata, Japan;

Toure, Saliou, Faculty of Science, Abidjan, Ivory Coast;

Tserpes, Nicholas A., Wayne State University;

Vanden Boss, Eugene L., Michigan State University;

Vander Brug, Gordon J., Calvin College;

Van Meter, Garrett Oliver II, University of Maryland;

Vineyard, James D., Laney College;

Voegele, Heinz S., University of British Columbia;

Walker, James R., DeVlieg Machine Company, Royal Oak, Michigan;

Wallen, Lawrence J., University of Michigan;

Wasserman, Arthur G., Harvard University;

Waterhouse, Richard N., Harvard Medical School, Boston, Massachusetts;

Wayment, Stanley Glen, Utah State University;

Wegener, Delano Paul, Ohio University;

Wehlen, Joseph A. Jr., Michigan State University;

Weiss, David M., Leesona-Moos Laboratories, Great Neck, New York;

Wessell, W. Roy, Argonne National Laboratory;

Weston, James H., University of Saskatchewan-Regina;

White, Albert G. Jr., Illinois State University;

White, Michael W., Iowa State University;

Whittemore, Alice S. (Mrs.), Hunter College (CUNY);

Wiancko, Bruce E., Westinghouse, Madison, Pennsylvania;

Wignall, Thomas K., Memorial University of Newfoundland;

Williams, Dolan W., Collins Radio Company, Richardson, Texas;

Williams, Michael Z., Colorado State University;

Williams, Ronald O., General Electric Company, Houston, Texas;

Wilson, David G., International Business Machines Corporation, Bethesda, Maryland;

Winthrop, Kenneth R., Bronx Community College (CUNY);

Wolkowisky, Jay H., University of Colorado;

Woo, Kai Y., University of California, Irvine;

Wood, June P., South Texas College;

Yeung, Shiu F., Virginia Polytechnic Institute; 
Yuan, Chi, Massachusetts Institute of Technology;

Yudewitz, Samuel, Yudewitz Company, Bronx, New York;

Zajic, Elizabeth S. (Mrs.), London Life Insurance, London, Ontario, Canada;

Zee, Yun-Cheng, California State College at Fullerton;

Zerger, Theodore, Kansas Wesleyan University;

Zilcher, Paul, Communications and Systems Incorporated, Falls Church, Virginia.

It was announced that the following persons were admitted to membership in accordance with reciprocity agreements with the various mathematical organizations named:

Australian Mathematical Society: Sav R. Harasymiv, Peter J. Lorimer, Gordon E. Wall, William Lee Steiger.

Union Matematica Argentina: Edgardo L. Fernandez, Emilio N. Fischman, Carlos A. Robledo.

Osterreichische Mathematische Gesellschaft: Wilfred Imrich.

Societe Mathematique de Belgium: Jacques V. Boel, Robert Dieschbourg, Robert R. G. Gemine, Joseph A. Thas.

London Mathematical Society: John H. E. Cohn, Godfrey L. Isaacs, W. B. Raymond Lickorish, Kim-Leon Lim, Robert McFadden, David Pager, Ronald Tiffen.

Indian Mathematical Society: Bankim C. Chatterjee, Narain D. Gupta, R. Rama Iyer, J. G. Krishna, Hari M. Srivastava.

Mathematical Society of Japan: Takayuki Furuta, Hitosi Hiramatu, Takashi Karube, Shuichiro Maeda, Takizo Minagawa, Isao Miyadera, Shinnosuke Oharu, Tosiyuku Tugue, Junzo Wada, Shinzo Watanabe.

Societe Mathematique de France: Baktavatsalou, Charles Boutang, Haim Brezis, Daragomir Z. Djokovic, Ivar S. Ekeland, Moshe Eytan, Michel Gatesoupe, Kohur. N. Gowrisankaran, Michel Metivier, Jean-Paul Pier, Michel Jean Pommiez, Hubert Tavernier.

Edinburgh Mathematical Society: Jane A. Burlak, Ivor M. H. Etherington.

Wiskundig Genootschap te Amsterdam: Fredericus J. M. Barning, Leonard Lips, Jan W. Nienhoys.

Svenska Matematikersamfundet: Leif Arkeryd, Gunnar Blow, Matts R. Essen, Anders R. Hyllengren, Sven Anders Tengstrand.

Deutsche Mathematiker Vereinigung: Othmar Baier, Heinz D. Dombrowski, Boro Doring, Bernhard Gramsch, Erhard Heil, Erwin K. H. Hoehn, Karl Heinz Mayer, Siegfried H. R. Pawelke, Wolfram Schwabhauser, Hanns J. Weinert.

Gesellschaft fur Angewandte Mathematik und Mechanik: Kurt Nixdorff, Franz Pittnauer.

Schweizerische Mathematische Gesellschaft: Urs Stammbach.

The following individuals were elected to membership as nominees of the respective institutional members:

University of Alabama: Peter M. Gibson.

Amherst College: David S. Johnson.

Angelo State College: Cass L. Archer, Ronald B. Harrist, Terry A. Watkins.

Auburn University: Eugene S. Ball, David A. Moe, David E. Powell.

Bowling Green State University: Jesse D. Parate Jr., Gary J. Sherman, Larry G. Williams.

Brandeis University: Daniel Crespin, Michael R. Gabel, Ronald A. Gove. Silvio 
Greco, Robin A. Hur, Ulrich Koschorke, Max A. Knus, Ralph W. Leighton, Ronald A. Shapiro, Roy C. Smith.

Brandon University: James W. Cotts.

Brock University: Joan Heinrichs, Eric R. Muller.

Brooklyn College (CUNY): Harvey I. Epstein.

California Institute of Technology: Peter W. Day, James L. Fisher, Curtis Greene, Lucien B. Levy, John C. Wells.

California State College of Hayward: Patricia M. Banks, Jonathan E. Schaff.

University of California, Berkeley: Christopher J. Allday, Kalman G. Bauner Jr., William A. Brock, Lowell A. Carmony, Ian F. Carlstrom, David R. Coar, Lewis P. Douglas, Richard W. Edsinger, Alain J. Etcheberry, Louis A. Feldman, Walter C. Frey, John M. Guckenheimer, Carlos E. Harle, Walter L. Hill, Richard B. Hodges, Roger E. Howe, Steven L. Jordan, Peter J. Kiernan, Rodger G. Knaus, Richard J. Laver, Matthew C. Lee, Ronald D. Levine, Karl J. Lindberg, Narendra L. Maria, Michael A. Minovitch, Barry R. Moyer, Zbigniew H. Nitecki, Peter B. Percell, Denris G. Pixton, Karel L. Prikry, Malayattil Rabindranathan, Robert D. Ridgon, Peter Rosmarin, Joseph E. Schlessinger, Brian Schmidt, Chester R. Schneider, Robert Schwarz, Mark J. Shensa, Roger A. Simons, Richard J. Thompson, John E. Wolfe.

University of California, Los Angeles: Leon D. Neidleman.

University of California, Riverside: James R. Diederich, Stanton P. Philipp, Leland E. Rogers, Joseph J. Sroka.

University of California, Santa Barbara: Donald G. Hartig, John A. Loustau, Paul T. Ma, Michael H. Powell, Roger P. Ware, Phyllis M. Zweig.

Calvin College: Julius J. Vande Kopple.

Catholic University of America: Minoru Hasegawa, G. Clinton Sornberger, Vernon E. Zander.

University of Chicago: Susanna S. Epp, Marvin J. Kohn, Roger D. Nussbaum.

Clarkson College of Technology: Donald B. Gormley, Eric K. Poysa, Carol Weisbrod. Clemson University: Richard B. Evans, David A. Ott, Arthur G. Sparks.

University of Colorado: Charles H. Brase, Ahmed N. Currim, George S. Donovan, Larry V. Ellis, Robert L. Etherton, Leonard J. Gallagher, Hugh B. Maynard, Richard A. Sanerib Jr., Michael G. Stone.

Colorado State University: Carl D. Meyer Jr.

College of the Holy Cross: Frank Petrella Jr., John J. Lynch.

Columbia University: Spencer J. Bloch, David A. Edwards, Daniel Feigin, James W. Hargrove Jr., Gregory T. Jackson, Garo Kiremidjian, Michael S. Katz, Beverly A. Klostergaard, Michele R. Linch, William H. Moll, Richard D. Mosak, Alexander Nagel, Bruce A. Olsen, Jane A. Piore, Eddie R. Williams, Tsu C. Wu.

University of Connecticut: Michael B. Gregory, King T. Kwok, Alexander Markons, Richard H. Wyman.

Dartmouth College: John C. Chipman, Charles D. Feustel.

University of Dayton: Robert E. Buck Jr.

East Carolina University: Paul W. Haggard, Katye O. Sowell, Robert M. Woodside. Emory University: Caulton L. Irwin.

Georgetown University: Donald R. Colner, Giuliano Gnugnoli.

George Washington University: Lawrence A. Lee, Richard C. Stewart.

Goucher College: Frank L. Friedman.

Humboldt State College: Roy W. Tucker.

Hunter College (CUNY): George G. Bertles, Harold H. Davitt, Michael A. Infranco. 
University of Illinois: Melvin F. Gardner, John B. Jacobs, Bhagat Singh, Emile Y. State, John D. White.

Illinois State University: James J. Hirstein, Kenneth R. Ori, Dale F. Perona.

University of Illinois at Chicago: Peter F. Ash, Peter S. Schlitz.

Institute for Advanced Study: Yakar I. Kannai.

University of Iowa: Michael R. Cullen, John P. Neuzil, David R. Scribner.

Iowa State University: William B. Johnson, Werner W. Shoultz.

Kansas State University: M. Poinsignon, Richard E. Propes, Gerald C. Schrag.

Kent State University: Grant E. Hagerty, Eunice B. Krinsky, Richard Arthur Shoop.

University of Kentucky: Donald Bennett, James J. Bowe, Grady L. Cantrell, Allen

K. Chelgren, Charles E. Cleaver, Rudy L. Curd, James G. Dobbins, Douglas P.

Elosser, Amy C. King, Georeg C. T. Kung, C. Bruce Myers, James D. Nelson, Henry B. Potoczny, Dennis H. Schnack, Clifford J. Swauger Jr., Nancy A. Winkelman.

Louisiana State University, Baton Rouge: Lloyd A. Blanchard, Willis J. Boruque Jr., Robert R. Butts, Samuel H. Cox Jr., Bill J. Dulin, Paul M. Eakin Jr., John R. Gilbert Jr., James R. Holub, Austin J. Lemoine, Yves Lequain, John D. McCharen, Thomas J. Smith, Charles P. Stegall Jr., Roger A. Waggoner, William W. Williams.

Macalester College: Robert L. Haar.

University of Maryland: John H. Javila Jr., John C. Burns, Raymond C. Cavanagh, Arrigo Cellina, Stephen R. Hilbert, Everett L. Lady, Michael B. Rosenzweig, Robert G. Voigt.

Massachusetts Institute of Technology: Robert F. Bergerou Jr., John H. Doles III, James D. Fabrey, Eric M. Friedlander, Elliot C. Gootman, Robert Gold, Arnold K. Griffith, Philip O. Koch, Charles G. Lange, James I. Lepowsky, Duncan B. Harris, Robert R. Kallman, Daniel B. Kotlow, Michael A. Machtey, William W. Roberts Jr., John W. Rosenthal, John C. Stillwell, Robert P. Walker, Gayn B. Winters.

University of Massachusetts: Chin-Hung Ching, Jonathan B. Skinner.

Memorial University of Newfoundland: Kanhayo L. Singh.

University of Michigan: James R. LaPalm.

University of Minnesota: David R. Adams, Leo J. Alex, Robert F. Anderson, Jerome L. Caldwell Jr., John H. Drew, Lawrence M. Gruman, Bradley W. Hempstead, Walter J. Hendricks, Emil F. Knapp. Robert W. Langer, Stephen A. McGrath, David G. Mutchler, Thomas Naughton, Jon W. Perry, Robert L. Raymond, Grenith J. Zimmerman.

University of Mississippi: Charles A. Brown, Jack D. Wilson.

University of Missouri at Rolla: Harmon C. Brown, Derald D. Rothmann, Darrell R. Thoman.

Montana State University: Leroy R. Amunrud, Oskar Feichtinger, Gilbert W. Nelson. University of Montreal: Pierre Decelles, Mohamed Djedour, Jacques A. DuBois, Gaston Giroux, Narendra K. Gobvil, Maurice Hervieux, Raymond J. LeBlanc, Gilles Pare.

Morehouse College: Kenneth J. Fagen, David L. Johnson, W. J. Morris.

New Mexico State University: Mohammed Bahauddin, Donald Cook, Joe L. Howard, Chiev Khus, Conrad K. McKnight.

University of New Mexico: Gail L. Carns, Charles N. Walter.

New York University-Courant: William M. Anderson, Carlos A. Berenstein, Peter E. Castro, Li-An Chen, Neil H. Fenichel, Michael Glass, William Goodhue, Nor- 
berto L. Kerzman, Hitoshi Kumano-Go, Luis J. Ledesma, John F. Ledlie, Norman J. Levitt, Roger Y. S. Lynn, Stephen A. Marion, Richard Alan Miller, Jeffrey B. Rauch, Niko Sauer, Arthur D. Snider, Joseph F. Sullivan, Kjell-Ove H. Widman.

North Carolina State University at Raleigh: Stephen H. Brown, Noal C. Harbertson, Gary D. Richardson.

University of North Carolina, Chapel Hill: Douglas M. Campbell, Andrew A. Blanchard, Susan W. Harris, Carolyn J. Hochanadel, Thomas C. Lominac, Richard K. Molnar, Stephen R. Swearingen.

North Texas State University: Edward W. Davenport, R. Don Lumley, Joel D. Price. Northwestern University: Shimshon Berkovits, Stephen D. Brown, Charles J. Cantor, William Erb Dietrich Jr., David S. Klatman, Ronald J. Webber.

University of Notre Dame: Joseph J. Bucuzzo, Chao-Kun Cheng, Alexander J. Hahn, Hsu Shih Ling, Ramesh K. Miglani, Takushiro Ochiai, Yi-Chuan Pan, Robert E. Solazzi.

Old Dominion College: Charlotte A. Cunningham, Carol L. Holley, Eileen A. Smith. Ohio University: Arthur E. Bukowski, S. Owen Burkinshaw.

Ohio Wesleyan University: Gregg W. Johnson.

Oregon State University: Donald R. Short Jr.

Pacific Lutheran University: Frank A. Hagen, Kenneth A. Lueder, Gary D. Peterson. University of Pittsburgh: Kenneth Schoen.

Polytechnic Institute of Brooklyn: Erwin Lutwak.

Portland State College: Ben E. Ermence.

Queens College (CUNY): Judy B. Peyer.

University of Rochester: Thomas B. Garner.

Rockefeller University: James K. Baker, Eugene M. Kleinberg.

Sacramento State College: Tae Woo Park, Jack F. Schlotthauer, Thomas E. Vollman. San Francisco State College: Mary E. Graham, Edith P. Mendez, Yee-Chung Bai-Yip Ying.

Seattle University: Lawrence J. Dickson, William R. Topp.

Shrimer College: Thomas L. Burgess, David M. James, Stewart C. Spyker.

University of the South: David P. Dyer, Robert M. Patterson.

University of South Carolina: Paul E. Bland.

Southern Illinois University: Elena B. Decima, Kent E. Foster, Samuel L. Lesseig. Southern Methodist University: Dovalle D. McElroy.

Southwestern at Memphis: Sandra S. Dunn, Sidney M. Webb, Edward T. Wright Jr. Stanford University: James T. Beale, Michael J. Beeson, Ronald E. Davis, Daniel E.

Erickson, Jack R. Gerson, Eugene L. Goldberg, Robert D. Gulliver, David H.

Halpert, David A. Hoffman, Alan T. Huckleberry, John E. Hutchinson, Lee K. Jones, Norman L. Kaplan, Richard P. Lacey, Bruce S. Lund, John C. Nash, Douglas J. Nelson, Stephen H. Polit, Michael R. Rolle, J. Richard Sajbel, Chester C. Seabury, Alan Smith, Duncan C. Thomas, Paul M. F. Tyu, Dennis J. Wildfogel.

College of Steubenville: Dennis A. McDonald.

State University of New York at Buffalo: Kenneth D. Elworthy, Robert D. Hofer, Karl

J. Klee, Sandra A. Monteferrante.

Suffolk University: Paul Ezust.

Sweet Briar College: John F. Collins Jr., Phillip M. Kannan.

Syracuse University: Ronald F. Barnes, Joseph M. Cavanaugh, B. Arthur Miller, Richard C. Orr, Sherman D. Riemenschneider, Richard M. Summerville. 
Texas Christian University: C. Wayne Mastin, Woodlea B. Sconyers, H. Edward Stone.

University of Texas: Jack B. Brown, Joseph E. Chance, Charles A. Coppin, John W. Hinrichsen, Gil C. Kim, John F. Lamb Jr., Richard D. Mauldin, Alvis E. McDonald, Merry G. McIlroy, Robert A. Northcutt, Charles E. Wilks.

University of Toledo: Jerry L. Edwards, William K. Hale.

University of Toronto: Jan W. Auer, Faith Y. Chao, Wai-Mee Ching, John C. Clements, Joseph Cunsolo, J. Gerard McPhail, John B. Walker, Timothy Walsh.

Tulane University: Thomas T. Bowman, John R. Martin, Jorge Martinez, Catherine L. Olsen, William B. Robinson, William R. Zame.

University of Tulsa: Carol S. Clark, Ellen F. Kilpatrick, John R. Sims.

University of Utah: James W. Cannon, Dennis D. Clayton, Nelson T. Dinerstein, Earl R. Heal, Paul W. Lewis, Stephen E. Newman.

Vanderbilt University: James L. Forde, Donald L. Greenwell, John Kinloch, James R. Gupton, Sarah E. Mullins, Kyle D. Wallace.

University of Virginia: Richard H. Bouldin, David C. Johnson, C. David Schweickart, James B. Wood.

University of Washington: Lee W. Erlebach.

Washington State University: James A. Beck, Joel D. Berman, Hang S. Chiang, Mary B. Ehlers, Kit Hanes, Norman L. Johnson, Richard G. Lee, Itrel E. Monroe, Roger A. Shorack, Douglas B. Smith.

Western Washington State College: Edwin S. Braithwaite, David B. Wagner.

University of Wisconsin: David D. Booth.

University of Wyoming: Ronald H. Dalla, Larry S. Johnson, Daryl Kreiling.

Xavier University: Robert C. Strunk.

Yeshiva University: Leon J. Ablon, Myron H. Bari, David M. Benovitz, William L. Bevilacqua, Michael Gartenberg, Wallace Goldberg, Jacob B. Gross, Beryl I. Shaw.

The institutions named below were elected to institutional membership:

New Haven College, West Haven, Connecticut Villanova University, Villanova, Pennsylvania Eastern Michigan University, Ypsilanti, Michigan

University of North Carolina, Charlotte, North Carolina

California State College, Los Angeles, California

Humboldt State College, Arcata, California

St. John's University, Jamaica, New York

The following committees have been established and the following Presidential appointments have been made:

Committee on a Research Expository Journal: M. Gerstenhaber, E. Hewitt, chairman, I. M. Singer.

Added to the AMS-IMS Committee on Translations: H. A. Antosiewicz and F. Browder to additional terms and E. Hewitt replacing V. Klee.

AMS-SIAM Joint Committee on Cooperation: Norman Levinson, Brockway McMillan, Barkley Rosser, chairman. 
Added to the Committee to Advise on the National Register: R. M. Thrall in place of R. F. Rinehart and A. B. Willcox in place of H. M. Gehman.

Committee to Select the Winner of the Bôcher Prize for 1969:

L. V. Ahlfors, chairman, Norman Levinson, Louis Nirenberg. Added to the Committee on Corporate Memberships: A. A. Albert in place of C. C. Hurd.

Added to the Joint Committee on Applied Mathematics: Calvin H. Wilcox.

Commission on the National Information System:

Representing the American Mathematical Society: F. Browder, J. Douglas, Jr., W. J. LeVeque, I. Niven, A. Rosenberg, G. L. Walker, D. Zelinsky

Representing the Mathematical Association of America: I. Niven

Temporarily representing the Association for Symbolic Logic: J. B. Rosser

Representing the Society for Industrial and Applied Mathematics: I. E. Block, J. W. Givens, W. J. Jameson, Jr., J. B. Rosser, D. L. Thomsen, F. J. Weyl

Temporarily representing The Institute of Mathematical Statistics: W. Hoeffding, S. Kotz

Temporarily representing the Association for Computing Machinery: J. F. Traub.

The following persons represented the Society at various functions as noted:

A. S. Galbraith at the inauguration of Albert Nathaniel Whiting as President of North Carolina College in Durham

J. W. Green at the inauguration of Charles Johnston Hitch as President of the University of California

Bernard Greenspan at the inauguration of J. Osborn Fuller as President of Fairleigh Dickinson University

Joseph J. Dennis at the inauguration of Frederick Corbet Davison as President of the University of Georgia

Guido Weiss at the Sesquicentennial Convocation of St. Louis University

H. Pearce Atkins at the inauguration of Luther W. White as President of Randolph-Macon College for Men.

The Secretary reported that the following persons had accepted invitations to give hour addresses: Robert Osserman and Robert Wolf at Riverside in November 1968; D. G. Higman and W.-Y. Hsiang at Evanston in November 1968; W. W. Boone, Edward 
Fadell, and François Treves at Chicago in April 1969; Tudor Ganea and David Harrison at Santa Cruz in April 1969.

The Council voted to reconsider an action of the Council of April 1968 [this Bulletin 74, (1968), 882] in the following form:

That Grothendieck's article on mathematics in North Vietnam [La vie mathématique en République Démocratique du Vietnam.] be printed in the NOTICES, subject only to the approval by Grothendieck of the translation and its publication. However, on a roll call vote the motion lost.

The Secretary called attention to the fact that the Society had received a legacy from the estate of Leroy P. Steele, consisting of capital in the amount of about $\$ 134,000$ and some additional income. The Executive Committee was asked to suggest uses for the money consistent with the suggestions in the will.

The Council established an ad hoc committee to prepare a pamphlet on the technique of expository writing.

The Council approved for presentation to the membership an amendment to the By-laws with the effect of eliminating the Committee on Printing and Publishing after 1971. It also approved a resolution setting the size of the Committee at three in 1969, two in 1970, and one in 1971. An effect of the resolution is that members of the Council by virtue of election to the Committee will serve the full terms to which they were elected.

The Council approved a committee recommendation that Raymond L. Wilder be invited to deliver the Gibbs Lecture of 1969.

The Nominating Committee proposed the name of Joseph B. Keller for election by the Council to represent the Society on the U.S. National Committee of the International Union of Theoretical and Applied Mechanics, an instrument of the National Research Council in the Division of Physical Sciences. The Council elected him to that position.

The report by the Nominating Committee of candidates for the 1969 election of Society officers was approved by the Council.

The candidates are as follows:

Vice President (one to be elected):

Secretary:

Associate Secretaries:

Treasurer:

Trustee:
H. S. M. Coxeter

Leo Zippin

Everett Pitcher

Leonard Gillman

O. G. Harrold, Jr.

W. T. Martin

Nathan Jacobson 
Bulletin Editorial Committee:

Proceedings Editorial Committee

Colloquium Editorial Committee:

Mathematical Reviews Editorial

Committee:

Mathematical Surveys Editorial

Committee:

Mathematics of Computation

Editorial Committee:

American Journal of Mathematics,

Representative:

Committee on Printing and Publishing: Members-at-large of the Council

(five to be elected):
Murray Gerstenhaber

Irving Glicksberg

George Mackey

F. W. Gehring

Michio Suzuki

Eugene Isaacson

Raoul Bott

Felix Browder

Hyman Bass, W.-L. Chow, Paul J. Cohen, Pierre Conner, Wallace Givens, Morris W. Hirsch, T. E. Hull, Victor Klee, Ray

A. Kunze, Louis Solomon.

The Committee to Monitor Problems in Communication proposed the creation of a plan for a National Information System. Approval in principle of a two-year design program was requested. It would follow a nine-month preliminary study already authorized by the Executive Committee and the Board of Trustees and in progress. It was the consensus of the Committee to Monitor Problems in Communication and in the Council that an extended span of time for consideration of the results of the nine-month preliminary survey, suggested by the Conference Board of the Mathematical Sciences and by the financial structure of the project, was intrinsically desirable. The request for approval in principle was received but no action was taken.

The Committee on a Research Expository Journal, charged "to study the desirability and the problems of publishing research expository papers in a new research expository journal or in the present journals of the Society," reported that "the Committee recommended that the Society continue to expand its policy of encouraging qualified mathematicians to write expository articles and survey-type book reviews and states its opinion that the best organ for these articles and reviews is the Bulletin." The Council approved.

The Council approved a proposal of the Joint Committee on Applied Mathematics for a Summer Seminar on "Mathematical Problems in the Geophysical Sciences" for the summer of 1969. 
The Council approved the offering over the signature of President Morrey of a letter to the Editor of Science. The letter was related to an extract from a speech by Dr. Donald Hornig, Presidential Science Adviser, published in Science, 161, p. 248 (July 19, 1968). The Council directed that the letter be published in the NOTICES. It may be found, together with the extract from the speech of Dr. Hornig, in the issue of October 1968.

The Business Meeting was convened by President Morrey at 4:35 P.M. on August 29, 1968 in the Union Theater on the University of Wisconsin campus. The number of persons in attendance increased as the meeting progressed, from about forty to about fifty-five.

The Secretary announced several actions of the Council of interest to the membership, including the action about inviting publication of the address of Professor Grothendieck.

Professor Nicholas D. Kazarinoff moved that the Secretary of the Society in the name of the American Mathematical Society invite Professor Grothendieck to submit his report [Mathematical Life in North Vietnam] to the NOTICES of the Society. The motion was seconded.

Objection was raised by Professor Saunders MacLane to consideration of the matter on the grounds that such a motion had twice been considered by the Council and twice had lost. The objection was overruled. It was established that, according to the By-laws, final action on the motion of Professor Kazarinoff could be taken only by unanimous consent or at a future meeting, with the full membership notified of the business in the call for the meeting. Unanimous consent was not forthcoming. The Society proceeded to the matter of directing the Secretary to notify the membership, in the call for the business meeting at the Annual Meeting of 1969, that the motion of Professor Kazarinoff would be on the agenda. In the course of the discussion Professor Kazarinoff proposed that copies of the report be distributed to the membership to assist them in deciding how they might vote.

On the motion to direct the Secretary, the vote was challenged. Procedural difficulties arose. During the discussion, the President entertained a motion for adjournment by Professor Alder. The motion was seconded and was passed at 5:14 P.M.

Bethlehem, Pennsylvania

Everett Pitcher Secretary

Paul Bateman Associate Secretary

URBANA, ILLINOIS 